\title{
Supersymmetric quantum mechanics in a first-order Dirac equation
}

\author{
Richard J. Hughes \\ Theoretical Division, Los Alamos National Laboratory, Los Alamos, New Mexico 87545 \\ V. Alan Kostelecký ${ }^{*}$ \\ Department of Physics, Indiana University, Bloomington, Indiana 47405 \\ Michael Martin Nieto ${ }^{\dagger}$ \\ Theoretical Division, Los Alamos National Laboratory, Los Alamos, New Mexico 87545 \\ and Niels Bohr Institutet, Blegdamsvej 17, 2100 København $\emptyset$, Denmark
}

(Received 18 April 1986)

\begin{abstract}
We demonstrate the realization of supersymmetric quantum mechanics in the standard first-order Dirac equation describing a massless Dirac particle in a magnetic field. This system is relevant to the integer quantum Hall effect. In obtaining the first-order supersymmetry, square-root operators are used and justified. A detailed discussion is also provided of the simpler problem of supersymmetry in the context of the relativistic Pauli Hamiltonian squared. In addition we discuss a realization of the superalgebra $\operatorname{osp}\left(\frac{1}{2}\right)$ obtained from this system.
\end{abstract}

\section{INTRODUCTION}

The startling discovery ${ }^{1}$ of the integer quantum Hall effect presented physicists with the possibilities of obtaining an improved value of the fine-structure constant and a standard of resistance at the quantum level. ${ }^{2}$ A theoretical explanation of the phenomenon has been advanced by Laughlin $^{3}$ and others. ${ }^{4,5}$ The system studied by Laughlin is that of nonrelativistic, spinless electrons confined to a plane with a uniform electric field in the plane and a uniform magnetic field perpendicular to the plane. The relevant Hamiltonian is the Pauli Hamiltonian and the solution of the eigenvalue problem is a series of Landau levels, all doubly degenerate except for the ground state.

This explanation can be viewed as involving the properties of the magnetic translation group in the plane. ${ }^{6-8}$ Laughlin's conclusions should therefore be unchanged by the introduction of electron spin. Indeed, it is now known that there are no relativistic corrections to Laughlin's explanation. 9,10

Many authors ${ }^{9-13}$ have investigated the normal quantum mechanics of an electron in a uniform magnetic field. These analyses can be pursued further in the context of supersymmetry in quantum mechanics. ${ }^{14,15}$ For example, if a nonrelativistic electron with a Pauli magnetic moment having a gyromagnetic ratio of two is placed in Laughlin's system, a supersymmetry occurs. ${ }^{16}$

In the Dirac theory a gyromagnetic ratio of two arises automatically. Further, as has recently been pointed out, ${ }^{17-19}$ the square of the Dirac Hamiltonian containing a uniform magnetic field coincides mathematically with the previously mentioned supersymmetry of the Pauli Hamiltonian. This supersymmetry is only manifest in the energy-squared spectrum since the quantum-mechanical algebra sqm(2) involves a second-order differential operator in its usual realization.

The above led us to ask if a supersymmetry could be found in the first-order Dirac formalism. In this work we elucidate our affirmative answer. ${ }^{19}$ We shall present the first example of supersymmetric quantum mechanics applied to the spectra obtained from the first-order Dirac Hamiltonian, rather than to spectra obtained from a second-order differential operator such as the Schrödinger Hamiltonian, the Pauli Hamiltonian, the Klein-Gordon equation, or the squared Dirac Hamiltonian.

We will work with a model Hamiltonian that describes massless Dirac fermions in a constant, uniform, magnetic field. The absence of an electric field may, in principle, be remedied by boosting the system. Also, although the massless limit is unphysical, the extension to the massive case is conceptually straightforward.

In Sec. II we establish our conventions, and we present the derivation of solutions to our model Hamiltonian. A discussion of the supersymmetry in the squared Dirac Hamiltonian, or ("second-order relativistic Pauli Hamiltonian") follows in Sec. III.

Section IV contains the major results. We explicitly construct matrix operators that yield a realization of supersymmetric quantum mechanics for our model firstorder Dirac Hamiltonian. Intuitively, the "square root" of the Dirac equation is thereby obtained.

The validity of using square-root operators is discussed in Sec. $\mathrm{V}$.

In Sec. VI magnetic translation symmetries for the Dirac Hamiltonian are discussed. Together with oscillator raising and lowering operators that appear in the Dirac Hamiltonian, we find that the generators of these symmetries may be used to form a set of operators closing into a superalgebra isomorphic to $\operatorname{osp}\left(\frac{1}{2}\right)$.

\section{THE MODEL AND ITS SOLUTIONS}

The minimally coupled Dirac equation for massless spinors is 


$$
\not D \Psi \equiv\left[\left(\partial_{t}-i e A_{t}\right) \gamma^{0}+(\partial+i e \mathbf{A}) \cdot \gamma\right] \Psi=0 .
$$

We choose to use the chiral representation of the Dirac $\gamma$ matrices:

$\gamma^{0}=\left(\begin{array}{cc}0 & -I \\ -I & 0\end{array}\right), \quad \gamma^{i}=\left[\begin{array}{cc}0 & \sigma^{i} \\ -\sigma^{i} & 0\end{array}\right), \quad i=1,2,3$,

where

$$
\begin{aligned}
& \sigma^{1}=\left(\begin{array}{ll}
0 & 1 \\
1 & 0
\end{array}\right), \quad \sigma^{2}=\left(\begin{array}{cc}
0 & -i \\
i & 0
\end{array}\right), \\
& \sigma^{3}=\left(\begin{array}{cc}
1 & 0 \\
0 & -1
\end{array}\right) .
\end{aligned}
$$

A constant, uniform, magnetic field can be described by

$$
A_{t}=0, A_{x}=-B y, \quad A_{y}=0, \quad A_{z}=0,
$$

giving $\mathbf{B}=\nabla \times \mathbf{A}=B \hat{z}$. Then, the particle will perform cyclotron motion in the $x-y$ plane. The operator $\not D$ is given by

$$
\not D=\gamma^{0} \partial / \partial t+\gamma \cdot \nabla-i e B y \gamma^{1} .
$$

We choose $e B>0$ for definiteness.

Solutions of (2.1) may be found via the Feynman $^{20,21}$-Gell-Mann ${ }^{21}$ method. ${ }^{10}$ First, the Dirac equation $(2.1)$ is multiplied by the Dirac operator $D$, yielding a second-order four-component equation:

$$
D D \Phi=0 \text {. }
$$

Equation (2.6) will have eight independent solutions $\Phi$. The solutions $\Psi$ of Eq. (2.1) are then given by

$$
\Psi=\not \Phi \Phi .
$$

The eight solutions so obtained are twice the number of independent ones. Equation (2.5) implies $D$ can be written as

$D=\left(\begin{array}{cccc}0 & 0 & -\partial_{t}+\partial_{z} & -D_{-} \\ 0 & 0 & +D_{+} & -\partial_{t}-\partial_{z} \\ -\partial_{t}-\partial_{z} & +D_{-} & 0 & 0 \\ -D_{+} & -\partial_{t}+\partial_{z} & 0 & 0\end{array}\right)$,

where

$$
D_{+} \equiv \partial / \partial x-i e B y+i \partial / \partial y
$$

and

$$
D_{-} \equiv D_{+}^{\dagger}=-\partial / \partial x+i e B y+i \partial / \partial y .
$$

Then, Eq. (2.6) may be written as

$$
\begin{aligned}
\not D \Phi \equiv & {\left[\left(\partial_{t}{ }^{2}-\partial_{x}{ }^{2}-\partial_{y}{ }^{2}-\partial_{z}{ }^{2}-2 i e B y \partial_{x}\right.\right.} \\
& \left.\left.+e^{2} B^{2} y^{2}\right) I+e B \sigma^{12}\right] \Phi \\
= & {\left[\left(\partial_{t}{ }^{2}-\partial_{z}{ }^{2}+D_{+} D_{-}\right) I+e B\left(I+\sigma^{12}\right)\right] \Phi=0 . }
\end{aligned}
$$

Here, $I$ is the $4 \times 4$ identity matrix and $\sigma^{12}=\frac{1}{2} i\left[\gamma^{1}, \gamma^{2}\right]$ is the $4 \times 4$ diagonal matrix with nonzero entries $(1,-1,1,-1)$.

In obtaining (2.11) we used the commutation relation

$$
\left[D_{-}, D_{+}\right]=2 e B \text {. }
$$

This indicates that $D_{+}$and $D_{-}$are unnormalized harmonic-oscillator raising and lowering operators, which will be of use to us in Secs. V and VI.

Equation (2.11) contains the gyromagnetic ratio of two. It may be solved by the ansatz

$\Phi_{n \epsilon \tau \sigma}(t, x, y, z) \equiv \exp \left[i \epsilon\left(-\omega_{n} t+k_{x} x+k_{z} z\right)\right] g_{n \epsilon \sigma}(y) \chi_{\tau \sigma}$,

where $\chi_{\tau \sigma}$ are chosen as the four, independent, constant, four-component eigenspinors of the matrices

$$
\gamma^{5}=\left(\begin{array}{ll}
I & \\
& -I
\end{array}\right)
$$

and $\sigma_{12}$, with

$$
\gamma^{5} \chi_{\tau \sigma}=\tau \chi_{\tau \sigma}
$$

and

$$
\sigma^{12} \chi_{\tau \sigma}=\sigma \chi_{\tau \sigma}
$$

where $\tau, \sigma= \pm 1$. The matrices $\gamma^{5}$ and $\sigma^{12}$ commute with each other and with the operator $D D$. Thus, the spinors $\chi_{\tau \sigma}$ have unity in the first through fourth rows, respectively, and zeros elsewhere.

The solutions (2.13) are also labeled by the sign $\epsilon$ of the energy, and by an integer $n \geq 0$. This integer arises because the functions $g_{n \in \sigma}(y)$ in Eq. (2.13) are found to be harmonic-oscillator functions, with centers displaced from the origin by an amount dependent upon the $x$ momentum, $k_{x}$ (Ref. 22).

A conserved, Hermitian, but non-positive-definite scalar product for any two solutions $\Phi$ and $\Phi^{\prime}$ of Eq. (2.11) is

$$
\left\langle\Phi \mid \Phi^{\prime}\right\rangle \equiv i \int d^{3} x \Phi^{*}(t, \mathbf{x}) \overleftrightarrow{\partial} / \partial t \Phi^{\prime}(t, \mathbf{x})
$$

With respect to this scalar product, a complete, orthogonal set of solutions to Eq. (2.11) for a given energy is given by $\Phi_{n \epsilon \tau \sigma}$ of Eq. (2.13), where

$$
\begin{aligned}
g_{n \epsilon \sigma}(y)= & \exp \left[-\frac{1}{2} e B\left(y-\epsilon k_{x} / e B\right)^{2}\right] \\
& \times H_{n-1 / 2-1 / 2 \sigma}\left((e B)^{1 / 2}\left(y-\epsilon k_{x} / e B\right)\right), \\
\omega_{n}=+( & \left(k_{z}^{2}+2 n e B\right)^{1 / 2},
\end{aligned}
$$

and $H_{n}$ are the Hermite polynomials. With this solution for $\Phi_{n \epsilon \tau \sigma}$ we find the identities

$$
\begin{gathered}
D_{+} \Phi_{n \epsilon \tau \sigma}(y)=-i(e B)^{1 / 2} \Phi_{n+1, \epsilon \tau \sigma}(y), \\
D_{-} \Phi_{n \epsilon \tau \sigma}(y)=+ \\
i(e B)^{1 / 2}(2 n-1-\sigma) \\
\times \Phi_{n-1, \epsilon \tau \sigma}(y) .
\end{gathered}
$$

Equation (2.11) is a system of four second-order differential equations that yields eight independent solutions, $\Phi_{n \epsilon \tau \sigma}$, for each value of the momenta $k_{x}$ and $k_{z}$. Further, the functional form of the solutions is independent of $\tau$. 
There is therefore a double degeneracy among the eight solutions, although they are independent because the spinors $\chi_{\tau \sigma}$ are orthogonal with respect to Eq. (2.17). Also, the energy is independent of $k_{x}$. This is the familiar Landau-level degeneracy ${ }^{22,23}$ and reflects the magnetic translation symmetry ${ }^{6,7}$ in the $x-y$ plane.

Proceeding with the Feynman-Gell-Mann method, we use Eq. (2.7) to obtain solutions of Eq. (2.1). Since Eq. (2.1) is a first-order differential equation, not all of the solutions $\Psi$ obtained from $\emptyset \Phi$ are linearly independent with respect to the conserved, Hermitian scalar product

$$
\begin{aligned}
\left\langle\Psi \mid \Psi^{\prime}\right\rangle & =\int d^{3} x \bar{\Psi}(t, \mathbf{x}) \gamma^{0} \Psi^{\prime}(t, \mathbf{x}) \\
& =\int d^{3} x \Psi^{\dagger}(t, \mathbf{x}) \Psi^{\prime}(t, \mathbf{x}) .
\end{aligned}
$$

Another aspect of this is that when we write Eq. (2.21) in the Hamiltonian form

$$
i \frac{\partial \Psi}{\partial t}=H_{D} \Psi
$$

where $H_{D}$ is the $4 \times 4$ matrix

$$
H_{D}=\left(\begin{array}{cc}
h_{D} & 0 \\
0 & -h_{D}
\end{array}\right) \text {, }
$$

and $h_{D}$ is the $2 \times 2$ matrix

$$
h_{D}=\left(\begin{array}{ll}
-i \partial / \partial z & i D_{-} \\
-i D_{+} & i \partial / \partial_{z}
\end{array}\right),
$$

we find that $\left[H_{D}, \gamma^{5}\right]=0$ but $\left[H_{D}, \sigma^{12}\right] \neq 0$. This means that the eigenvalues $\sigma$ of $\sigma^{12}$ cannot be used to label the solutions of $H_{D}$. In fact, this can be viewed as the origin of there being only four instead of eight independent solutions $\Psi$.

This overcompleteness is explicitly seen by calculating

$$
\not D \Phi_{n \epsilon \tau-}=\frac{\tau \epsilon\left(\omega_{n}+\tau k_{z}\right)}{(e B)^{1 / 2}} \not D \Phi_{n \epsilon \tau+} .
$$

Equation (2.25) shows that $\sigma$ is not an independent quantum number in the Dirac space, whereas $\epsilon$ and $\tau$ are. Defining

$Y_{\epsilon}=(e B)^{1 / 2}\left(y-\epsilon k_{x} / e B\right)$,

$G_{\epsilon}=\exp \left[+i \epsilon\left(-\omega_{n} t+k_{x} x+k_{z} z\right)\right] \exp \left(-\frac{1}{2} Y_{\epsilon}{ }^{2}\right)$,

the four independent unnormalized solutions are $(\epsilon= \pm 1)$

$\Psi_{n \epsilon+}=\not D \Phi_{n \epsilon++}=G_{\epsilon}\left(\begin{array}{c}0 \\ 0 \\ i \epsilon\left(\omega_{n}-k_{z}\right) H_{n-1}\left(Y_{\epsilon}\right) \\ i(e B)^{1 / 2} H_{n}\left(Y_{\epsilon}\right)\end{array}\right)$

$\Psi_{n \epsilon-}=\not D \Phi_{n \epsilon-+}=G_{\epsilon}\left(\begin{array}{c}i \epsilon\left(\omega_{n}+k_{z}\right) H_{n-1}\left(Y_{\epsilon}\right) \\ -i(e B)^{1 / 2} H_{n}\left(Y_{\epsilon}\right) \\ 0 \\ 0\end{array}\right)$,

where the $H_{n}$ are the Hermite polynomials $\left(H_{-1} \equiv 0\right)$.

By now defining

$$
\begin{aligned}
f_{n \epsilon}= & \frac{\exp \left(i \epsilon k_{x} x\right)}{(2 \pi)^{1 / 2}}\left(\frac{(e B)^{1 / 2}}{\pi^{1 / 2} 2^{n} n !}\right)^{1 / 2} \\
& \times \exp \left(-\frac{1}{2} Y_{\epsilon}{ }^{2}\right) H_{n}\left(Y_{\epsilon}\right),
\end{aligned}
$$

with the properties

$$
\begin{aligned}
& \int f_{n^{\prime} \epsilon}^{*} f_{n \epsilon} d x d y=\delta_{n^{\prime} n} \delta\left(\epsilon\left(k-k^{\prime}\right)\right), \\
& D_{ \pm} f_{n \epsilon}=\mp i[(e B)(2 n+1 \pm 1)]^{1 / 2} f_{n \pm 1, \epsilon}
\end{aligned}
$$

we can simply obtain the eigenstates normalized to

$$
\delta\left(\epsilon\left(k_{x}-k_{x}^{\prime}\right)\right) \delta\left(\epsilon\left(k_{z}-k_{z}^{\prime}\right)\right) .
$$

They are

$$
\begin{aligned}
& \lim _{n \rightarrow 0} \frac{f_{n}}{n^{1 / 2}} \equiv 0, \\
& \Psi_{n \epsilon+}=N_{\epsilon+}\left(\begin{array}{c}
\epsilon\left(\omega_{n}-k_{z}\right)(2 n)^{-1 / 2} f_{n-1, \epsilon} \\
(e B)^{1 / 2} f_{n, \epsilon}
\end{array}\right], \\
& \Psi_{n \epsilon-}=N_{\epsilon-}\left(\begin{array}{c}
\epsilon\left(\omega_{n}+k_{z}\right)(2 n)^{-1 / 2} f_{n-1, \epsilon} \\
-(e B)^{1 / 2} f_{n, \epsilon} \\
0 \\
0
\end{array}\right), \\
& N_{\epsilon \pm}=\frac{\exp \left[i \epsilon\left(-\omega_{n} t+k_{z} z\right)\right]}{(2 \pi)^{1 / 2}} \\
& \quad \times\left[e B+\left(1-\delta_{n 0}\right)\left(\omega_{n} \mp k_{z}\right)\right] 2^{-1 / 2} .
\end{aligned}
$$

\section{SUPERSYMMETRY AND THE RELATIVISTIC PAULI HAMILTONIAN SQUARED}

A quantum-mechanical system is said to be supersymmetric $^{14-16}$ if there exist operators $Q_{i}, i=1, \ldots, N$, that commute with the Hamiltonian $H_{\mathrm{SS}}$ and that anticommute to generate the Hamiltonian

$$
\begin{aligned}
& {\left[Q_{i}, H_{\mathrm{SS}}\right]=0,} \\
& \left\{Q_{i}, Q_{j}\right\}=\delta_{i j} H_{\mathrm{SS}} .
\end{aligned}
$$

We refer to the superalgebra (3.1) as $\operatorname{sqm}(N)$.

In this paper, we shall restrict our attention to the superalgebra $\operatorname{sqm}(2)$, which has two anticommuting generators. Furthermore, we shall work with matrices $Q$ and $Q^{\dagger}$, defined by

$$
\begin{aligned}
& Q=\frac{1}{\sqrt{2}}\left(Q_{1}+i Q_{2}\right), \\
& Q^{\dagger}=\frac{1}{\sqrt{2}}\left(Q_{1}-i Q_{2}\right)
\end{aligned}
$$

Then, the superalgebra $\operatorname{sqm}(2)$ may be written as 


$$
\begin{aligned}
& {\left[Q, H_{\mathrm{SS}}\right]=\left[Q^{\dagger}, H_{\mathrm{SS}}\right]=0} \\
& \{Q, Q\}=\left\{Q^{\dagger}, Q^{\dagger}\right\}=0 \\
& \left\{Q, Q^{\dagger}\right\}=H_{\mathrm{SS}}
\end{aligned}
$$

$H_{\text {SS }}$ has an eigenspectrum with a set of pairwise degenerate "boson" and "fermion" levels, and with a single "boson" ground state. The operator $Q$ takes a fermionic eigenstate $|F\rangle$ into a bosonic eigenstate $|B\rangle$. The reverse transformation is done by $Q^{\dagger}$. In equation form we have

$$
\begin{aligned}
& Q|F\rangle=E^{1 / 2}|B\rangle, \\
& Q^{\dagger}|B\rangle=E^{1 / 2}|F\rangle,
\end{aligned}
$$

where $E$ is the common eigenenergy of $|B\rangle$ and $|F\rangle$. For unbroken supersymmetry, both $Q$ and $Q^{\dagger}$ annihilate the ground state $|0\rangle$ :

$$
Q|0\rangle=Q^{\dagger}|0\rangle=0 \text {. }
$$

This and the superalgebra (3.3) imply that the eigenenergy of the ground state is necessarily zero, i.e.,

$$
H_{\text {SS }}|0\rangle=0 \text {. }
$$

Note that the superalgebra (3.3) and Eq. (3.6) mean that $H_{\mathrm{SS}}$ has eigenvalues that all must be greater than or equal to zero.

Let us turn to the role of the superalgebra sqm(2) in the system described by the relativistic Pauli Hamiltonian squared,

$$
\left(H^{2}\right)_{\mathrm{RP}}=\left(H_{D}\right)^{2},
$$

where $H_{D}$ is given by Eqs. (2.23) and (2.24). There is a relationship between this system and the nonrelativistic supersymmetric harmonic oscillator. ${ }^{17}$ Defining

$$
\begin{aligned}
& Q_{\mathrm{RP}}=\left(\begin{array}{cc}
0 & 0 \\
h_{D} & 0
\end{array}\right), \\
& Q_{\mathrm{RP}}^{\dagger}=\left(\begin{array}{cc}
0 & h_{D} \\
0 & 0
\end{array}\right),
\end{aligned}
$$

we find that

$$
\left\{Q_{\mathrm{RP}}, Q_{\mathrm{RP}}^{\dagger}\right\}=\left(H^{2}\right)_{\mathrm{RP}} .
$$

The operators $Q_{\mathrm{RP}}$ and $Q_{\mathrm{RP}}^{\dagger}$ satisfy the sqm(2) algebra in Eq. (3.3). Now,

$$
\left\{Q_{\mathrm{RP}}, \gamma^{5}\right\}=\left\{Q_{\mathrm{RP}}^{\dagger}, \gamma^{5}\right\}=0
$$

Thus, the supersymmetry generators $Q_{\mathrm{RP}}$ and $Q_{\mathrm{RP}}^{\dagger}$ act on the eigenvectors of $\left(\mathrm{H}^{2}\right)_{\mathrm{RP}}$ to change the eigenvalue $\tau$ of $\gamma^{5}$ and also to change the sign $\epsilon$ of the Dirac energy. However, since the eigenvalues of $\left(H^{2}\right)_{R P}$ are energies squared, $Q_{\mathrm{RP}}$ and $Q_{\mathrm{RP}}^{\dagger}$ interrelate degenerate eigenstates in the relativistic Pauli system, as expected.

It is also interesting to note that the Dirac Hamiltonian may be written as $^{24}$

$$
H_{D}=\gamma^{0}\left(Q_{\mathrm{RP}}^{\dagger}-Q_{\mathrm{RP}}\right) .
$$

A relationship of this type might have been expected, since both $Q_{\mathrm{RP}}$ and $H_{D}$ are "square roots" of $\left(H^{2}\right)_{\mathrm{RP}}$.

We remark that what we are calling the "ground state" of $\left(H^{2}\right)_{R P}$ is really an infinite set of levels, labeled by the continuous variable $k_{z}$, as may be seen from Eqs. (2.13), (2.18), and (2.19). The "ground state" energy is $\omega_{0}{ }^{2}=k_{z}{ }^{2}$, which is zero only when $k_{z}=0$. Therefore, from Eqs. (3.5) and (3.6) and the associated discussion, the $\operatorname{sqm}(2)$ supersymmetry of Eq. (3.9) is an unbroken symmetry of the second-order relativistic Pauli system only in the limit $k_{z} \rightarrow 0$. Interestingly, this limit is in force under the experimental conditions of the quantized Hall effect.

The breaking of the supersymmetry for $k_{z} \neq 0$ may be explicitly seen by acting with $Q_{\mathrm{RP}}$ of Eq. (3.8) on the ground-state eigenvector of Eqs. (2.13), (2.18), and (2.19). We find

$$
Q_{\mathrm{RP}} \Phi_{0 \epsilon \tau-}(0, x, y, z)=-\epsilon k_{z} \Phi_{0 \epsilon--}(0, x, y, z) .
$$

Although part of the ground-state eigenvector, specifically $\Phi_{0 \epsilon+-}$, is indeed annihilated by $Q_{\mathrm{RP}}$, the remainder $\Phi_{0 \epsilon--}$ is not.

\section{SUPERSYMMETRY AND THE DIRAC HAMILTONIAN}

In this section, we investigate the existence of supersymmetry for particles in the system described by the first-order Dirac Hamiltonian $H_{D}$, Eqs. (2.22) and (2.23). Inspired by the results of the last section, we look for operators $Q_{D}$ and $Q_{D}^{\dagger}$ that are square roots of $H_{D}$.

The easiest way to obtain such $Q_{D}$ and $Q_{D}^{+}$is first to find the diagonal form $H_{D}^{\prime}$ of $H_{D}$. To see that the diagonal form $H_{D}^{\prime}$ can be constructed, note that $\left(H_{D}\right)^{2}=\left(H^{2}\right)_{\mathrm{RP}}$ is diagonal. Therefore, $H_{D}^{\prime}$ has entries that are one of the square roots of the diagonal entries of $\left(H^{2}\right)_{\mathrm{RP}}$. Then, after one obtains the supersymmetry operators $Q_{D}^{\prime}$ and $Q_{D}^{\prime}$ from $H_{D}^{\prime}$, they must be converted to the $H_{D}$ basis. The Foldy-Wouthuysen ${ }^{25}(\mathrm{FW})$ transformation of $H_{D}$ into $H_{D}^{\prime}$ is the straightforward way of doing this. We use the FW transformation

$$
H_{D}^{\prime}=U^{-1} H_{D} U
$$

where

$$
U=\exp \left(\frac{1}{2} \gamma^{3} \Pi_{\perp} \cdot \gamma_{\perp} \theta\right)
$$

In Eq. (4.2),

$$
\begin{aligned}
& \boldsymbol{\Pi}_{\perp} \cdot \gamma_{\perp}=\Pi^{1} \gamma^{1}+\Pi^{2} \gamma^{2}, \\
& \Pi^{1}=k_{x}-e B y, \\
& \Pi^{2}=k_{y} .
\end{aligned}
$$

Further defining

$$
\begin{aligned}
& \tan \xi \theta=\frac{\xi}{k_{z}}, \\
& \xi^{2}=-\left(\boldsymbol{\Pi}_{\perp} \cdot \gamma_{\perp}\right)^{2},
\end{aligned}
$$

Eqs. (4.1) and (4.2) can be combined to write $H_{D}^{\prime}$ as 


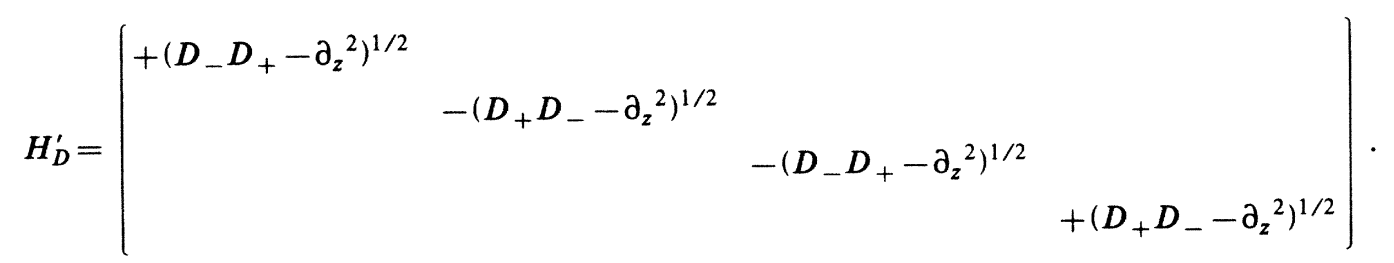

In the unbroken supersymmetry case we are interested in (Ref. 26 ), $k_{z}=0$ so that

$$
H_{D}^{\prime}\left(k_{z}=0\right)=\left(\begin{array}{llll}
\sqrt{D_{-} D_{+}} & & & \\
& -\sqrt{D_{+} D_{-}} & \\
& & -\sqrt{D_{-} D_{+}} & \\
& & \sqrt{D_{+} D_{-}}
\end{array}\right) .
$$

The spectrum of $H_{D}^{\prime}\left(k_{z}=0\right)$ is composed of four pieces. Each of them is associated with one of the operators that is on the diagonal in Eq. (4.6). Starting on the upper lefthand corner and going to the bottom right-hand corner, the four operators correspond to (i) positive energy and $e \mathbf{B} \cdot \boldsymbol{\sigma}>0$, (ii) negative energy and $e \mathbf{B} \cdot \boldsymbol{\sigma}>0$, (iii) negative energy and $e \mathbf{B} \cdot \boldsymbol{\sigma}<0$, and (iv) positive energy and $e \mathbf{B} \cdot \boldsymbol{\sigma}<0$. However, the supersymmetric generators of sqm(2) link one bosonic spectrum to one fermionic spectrum. Thus, only two of these four spectra can be accommodated simultaneously. We shall now discuss the different possible choices allowed by Eq. (4.6).

We start by constructing the supersymmetric quantum mechanics relating the two positive-energy spectra. The supersymmetric generators may be written

$$
Q_{D}^{\prime}=\left(\begin{array}{ccc}
0 & 0 & \sqrt{D_{-}} \\
& 0 & 0 \\
0 & & 0
\end{array}\right)
$$

and

$$
Q_{D}^{\prime \dagger}=\left(\begin{array}{ccc}
0 & & 0 \\
0 & 0 & \\
\sqrt{D_{+}} & 0 & 0
\end{array}\right) .
$$

These operators indeed generate the positive-energy spectra, since

$$
\begin{aligned}
\left\{Q_{D}^{\prime}, Q_{D}^{\prime \dagger}\right\} & =H_{D}^{\prime}\left(k_{z}=0, \epsilon=+1\right) \\
& =\left(\begin{array}{llll}
\sqrt{D_{-} D_{+}} & & \\
& 0 & & \\
& & 0 & \\
& & & \sqrt{D_{+} D_{-}}
\end{array}\right) .
\end{aligned}
$$

Next, we consider the superalgebra linking the negative-energy states of Eq. (4.6). In Sec. III we noted that a Hamiltonian associated with the superalgebra sqm(2) necessarily has positive or zero eigenvalues. Therefore, the negative-energy states are not linked by $\operatorname{sqm}(2)$. If we take

$$
\widetilde{Q}_{D}^{\prime}=\left(\begin{array}{ccc}
0 & & 0 \\
0 & \sqrt{D_{-}} & \\
0 & 0 & 0
\end{array}\right)
$$

and

$$
\widetilde{Q}_{D}^{\prime \dagger}=\left(\begin{array}{ccc}
0 & 0 & 0 \\
& \sqrt{D_{+}} & 0 \\
0 & & 0
\end{array}\right),
$$

then we find

$$
\begin{aligned}
\left\{\widetilde{Q}_{D}^{\prime}, \widetilde{Q}_{D}^{\prime \dagger}\right\} & =-H_{D}^{\prime}\left(k_{z}=0, \epsilon=-1\right) \\
& =\left(\begin{array}{llll}
0 & & \\
& \sqrt{D_{+} D_{-}} & & \\
& & \sqrt{D_{-} D_{+}} & \\
& & & 0
\end{array}\right) .
\end{aligned}
$$

Except for the minus sign in front of the Hamiltonian, this superalgebra obeys the commutation relations of sqm(2) given in Eq. (3.3). The relationship of this superalgebra to $\operatorname{sqm}(2)$ is similar to the relationship between the compact algebra so(3) and the noncompact algebra so $(2,1)$.

Then, since

$$
H_{D}^{\prime}\left(k_{z}=0\right)=\left\{Q_{D}^{\prime}, Q_{D}^{\prime \dagger}\right\}-\left\{\widetilde{Q}_{D}^{\prime}, \widetilde{Q}_{D}^{\prime \dagger}\right\},
$$

we also have that

$$
H_{D}\left(k_{z}=0\right)=\left\{Q_{D}, Q_{D}^{\dagger}\right\}-\left\{\widetilde{Q}_{D}, \widetilde{Q}_{D}^{\dagger}\right\},
$$

where

$$
\begin{aligned}
& Q_{D}=U Q_{D}^{\prime} U^{-1}, \\
& \widetilde{Q}_{D}=U \widetilde{Q}_{D}^{\prime} U^{-1} .
\end{aligned}
$$

One can also construct algebras linking the positiveand negative-energy spectra of Eq. (4.6). For example, defining

$$
X_{D}^{\prime}=\left(\begin{array}{ccc}
0 & \sqrt{D_{-}} & 0 \\
0 & 0 & \\
0 & & 0
\end{array}\right),
$$


and

$$
X_{D}^{\prime \dagger}=\left(\begin{array}{ccc}
0 & 0 & 0 \\
\sqrt{D_{+}} & 0 & \\
0 & & 0
\end{array}\right),
$$

we find

$$
\begin{aligned}
{\left[X_{D}^{\prime}, X_{D}^{\prime \dagger}\right] } & =H_{D}^{\prime}\left(k_{z}=0, \tau=+1\right) \\
& =\left(\begin{array}{llll}
\sqrt{D_{-} D_{+}} & & \\
& -\sqrt{D_{+} D_{-}} & \\
& 0 & \\
& & 0
\end{array}\right) .
\end{aligned}
$$

However, this algebra is not a superalgebra, since $X_{D}^{\prime}$ and $X_{D}^{\prime \dagger}$ commute to yield $H_{D}^{\prime}\left(k_{z}=0, \tau=+1\right)$. Furthermore, $X_{D}^{\prime}$ and $X_{D}^{\prime \dagger}$ are not constants of the motion.

Similarly one can construct algebras between any positive-energy spectrum and any negative-energy one. However, since these, too, are not superalgebras, we shall not consider them further, as our goal here is to investigate the role of supersymmetry in the Dirac equation.

\section{SQUARE-ROOT OPERATORS}

We now commute on the meaning of the square roots we have used in the operators of Sec. IV.

The square root in the FW Hamiltonian operators of Eqs. (4.5) and (4.6) is not unusual. It is a standard result when diagonalizing a Dirac Hamiltonian. ${ }^{25}$ The Dirac Hamiltonian itself has units of energy. Because of the Lorentz invariance of the problem, it will be represented by the square root of a momentum-squared term plus a mass-squared term. This is the same fact that led Dirac to his algebra as a device for taking the "square root" of the Klein-Gordon second-order operators.

Formally, since the sign of the square root is determined as positive (the overall sign coming from the diagonal Dirac matrix), its use can be justified in terms of power-series expansions. ${ }^{27}$ However, a transparent justification is that these operators can be defined operationally. They can be written in terms of the eigenstates we have defined previously.

Explicitly, given the diagonal FW eigenstates and eigenvalues, one can define the operators as diagonal matrices whose entries are the eigenvalues in the row column defined by particular eigenvectors.

The definitions of $\left(D_{-}\right)^{1 / 2}$ and $\left(D_{+}\right)^{1 / 2}$ at first appear less obvious. However, with the results of Sec. II they follow similarly. The key is the observation, from Eq. (2.12), that $D_{-}$and $D_{+}$are harmonic-oscillator lowering and raising operators. Thus, even though they are not Hermitian, their square roots can be defined in terms of coherent states.

Using the normed forms

$$
a=D_{-} / \sqrt{2 e B}, a^{\dagger}=D_{+} / \sqrt{2 e B},
$$

one has for coherent states $|\alpha\rangle$ that $^{28}$

$$
a|\alpha\rangle=\alpha|\alpha\rangle,\langle\alpha| a^{\dagger}=\langle\alpha| \alpha^{*} .
$$

Here, $\alpha$ is a complex number

$$
\alpha=\alpha_{1}+i \alpha_{2} \equiv \rho \exp (i \theta),
$$

with $0 \leq \arg \alpha<2 \pi$ and $-2 \pi<\arg \alpha^{*} \leq 0$. Thus,

$$
\begin{aligned}
& \left\langle\beta\left|a^{1 / 2}\right| \alpha\right\rangle=\alpha^{1 / 2}\langle\beta \mid \alpha\rangle, \\
& \left\langle\beta\left|\left(a^{\dagger}\right)^{1 / 2}\right| \alpha\right\rangle=\left(\beta^{*}\right)^{1 / 2}\langle\beta \mid \alpha\rangle, \\
& \langle\beta \mid \alpha\rangle=\exp \left(\beta^{*} \alpha^{-1 / 2}|\alpha|^{2}-\frac{1}{2}|\beta|^{2}\right),
\end{aligned}
$$

where $\arg \left(\alpha^{1 / 2}\right) \equiv \theta / 2$.

Therefore, as with the FW Hamiltonian, these square roots can be defined as matrices in the coherent-state basis. Their product can be written by using the resolution of the identity

$$
I=\frac{1}{\pi} \int_{-\infty}^{\infty}(d \operatorname{Re} \alpha)(d \operatorname{Im} \alpha)|\alpha\rangle\langle\alpha| \text {. }
$$

\section{MAGNETIC TRANSLATION SYMMETRIES AND osp ( $\left.\frac{1}{2}\right)$}

The Dirac Hamiltonian of Eq. (2.23) is translationally invariant in $x$. Further, up to an $x$-dependent gauge transformation, it is also translationally invariant in $y$. There are two oscillator raising and lowering operators ${ }^{29}$ which can generate these symmetry transformations. They are

$$
b \equiv \frac{-\partial}{\partial y}+i \frac{\partial}{\partial x}+i e B x
$$

and

$$
b^{\dagger}=\frac{\partial}{\partial y}+i \frac{\partial}{\partial x}-i e B x
$$

with

$$
\left[b, H_{D}\right]=\left[b^{\dagger}, H_{D}\right]=0,
$$

and

$$
\left[b, b^{\dagger}\right]=2 e B
$$

This implies that the Landau-level degeneracy is $(e B / 2 \pi)$ levels per unit area in the $x-y$ plane. The generators $Q_{\mathrm{RP}}$ and $Q_{\mathrm{RP}}^{\dagger}$ of Eq. (3.8) are independent of these symmetries since

$$
\left\{b, Q_{\mathrm{RP}}\right\}=\left\{b^{\dagger}, Q_{\mathrm{RP}}\right\}=\left\{b, Q_{\mathrm{RP}}^{\dagger}\right\}=\left\{b^{\dagger}, Q_{\mathrm{RP}}^{\dagger}\right\}=0 .
$$

Laughlin's explanation ${ }^{3}$ of the nonrelativistic quantized Hall effect uses these symmetries of the problem. $\mathrm{He}$ shows that the center of the harmonic-oscillator (Landaulevel) wave functions is moved in the $y$ direction by an $x$ dependent gauge transformation. However, an electric field can be considered as an adiabatic interpolation between two such gauge-transformed systems. Therefore, the charge transfer and the Hall current may be easily calculated.

With this physical insight in mind, we wish to present a superalgebra containing $b, b^{\dagger}$ and the raising and lowering operators $D$ and $D^{\dagger}$ of Eqs. (2.9) and (2.10). First, define the normalized operators $a_{i}$ and $a_{i}^{\dagger}, i=1,2$, as 


$$
\begin{aligned}
& a_{1}=\frac{1}{\sqrt{2 e B}} D_{-}, \\
& a_{1}^{\dagger}=\frac{1}{\sqrt{2 e B}} D_{+}, \\
& a_{2}=\frac{1}{\sqrt{2 e B}} b, \\
& a_{2}^{\dagger}=\frac{1}{\sqrt{2 e B}} b^{\dagger} .
\end{aligned}
$$

This gives

$$
\left[a_{i}, a_{j}^{\dagger}\right]=\delta_{i j} \text {. }
$$

Now define the operators

$$
\begin{aligned}
& K_{0}=\frac{1}{2} \sum_{i=1}^{2}\left(a_{i}^{\dagger} a_{i}+\frac{1}{2}\right) \\
& K_{+}=\frac{1}{2} \sum_{i=1}^{2} a_{i}^{\dagger} a_{i}^{\dagger} \\
& K_{-}=\frac{1}{2} \sum_{i=1}^{2} a_{i} a_{i} \\
& F_{+}=\frac{1}{2} \sum_{i=1}^{2} a_{i}^{\dagger} \sigma_{i} \\
& F_{-}=\frac{1}{2} \sum_{i=1}^{2} a_{i} \sigma_{i}
\end{aligned}
$$

where the Pauli matrices $\sigma_{1}$ and $\sigma_{2}$ are defined in Eq. (2.3).

The five operators of Eq. (6.8) generate the superalgebra $\operatorname{osp}\left(\frac{1}{2}\right)$ given by

$$
\begin{aligned}
& {\left[K_{0}, K_{ \pm}\right]= \pm K_{ \pm},\left[K_{+}, K_{-}\right]=-2 K_{0},} \\
& {\left[K_{0}, F_{ \pm}\right]= \pm \frac{1}{2} F_{ \pm},} \\
& {\left[K_{ \pm}, F_{ \pm}\right]=0, \quad\left[K_{ \pm}, F_{+}^{-}\right]=-F_{ \pm},} \\
& \left\{F_{ \pm}, F_{ \pm}\right\}=K_{ \pm}, \quad\left\{F_{+}, F_{-}\right\}=K_{0} .
\end{aligned}
$$

In Ref. 30, a physically related realization of $\operatorname{osp}\left(\frac{1}{2}\right)$ is given.

\section{ACKNOWLEDGMENTS}

R.J.H. thanks the California Institute of Technology for hospitality. V.A.K. enjoyed the hospitality of the Aspen Center for Physics, while part of this work was completed. The authors acknowledge the support of the United States Department of Energy under Contract No. W7405-ENG-36. V.A.K. also acknowledges support of the United States Department of Energy under Contract No. DE-AC02-84ER40125, Task B.
*Address during calendar year 1986: Theory Division, CERN, 1211 Genève 23, Switzerland.

${ }^{\dagger}$ Address during academic year 1985-1986: Niels Bohr Institutet.

${ }^{1}$ K. von Klitzing, G. Dorda, and M. Pepper, Phys. Rev. Lett. 45, 494 (1980).

${ }^{2}$ For overviews, see, for example, H. C. Stormer and D. C. Tsui, Science 220, 1241 (1983); M. E. Cage and S. M. Girvin, Comments Solid State Phys. 11, 47 (1983).

${ }^{3}$ R. B. Laughlin, Phys. Rev. B 23, 5632 (1981).

${ }^{4}$ B. I. Halperin, Phys. Rev. B 25, 2185 (1982).

5J. E. Avron and R. Seiler, Phys. Rev. Lett. 54, 259 (1985).

${ }^{6}$ E. Brown, Phys. Rev. 133, A1038 (1964).

7J. Zak, Phys. Rev. 134, A1602 (1964).

${ }^{8}$ R. Peierls, Z. Phys. 80, 763 (1933); H. Weyl, The Theory of Groups and Quantum Mechanics (Dover, New York, 1931), p. 272.

${ }^{9}$ A. H. MacDonald, Phys. Rev. B 28, 2235 (1983).

${ }^{10}$ M. M. Nieto and P. L. Taylor, Am. J. Phys. 53, 234 (1985).

${ }^{11}$ I. I. Rabi, Z. Phys. 49, 507 (1928); M. H. Johnson and B. A. Lippmann, Phys. Rev. 76, 828 (1949).

${ }^{12}$ H. Bacry, Ph. Combe, and J. L. Richard, Nuovo Cimento A 67, 267 (1970)

13J. Beckers and V. Hussin, J. Math. Phys. (N.Y.) 24, 1286 (1983).

${ }^{14}$ E. Witten, Nucl. Phys. B185, 513 (1982); P. Salomonson and J. W. van Holten, ibid. B196, 509 (1982); F. Cooper and B. Freedman, Ann. Phys. (N.Y.) 146, 262 (1983); F. Cooper, B. Freedman, and C. M. Bender, Nucl. Phys. B219, 61 (1983); D. Lancaster, Nuovo Cimento A 79, 28 (1984); M. M. Nieto, Phys. Lett. 145B, 208 (1984).
${ }^{15}$ Proposed physical applications are discussed in L. F. Urrutia and E. Hernandez, Phys. Rev. Lett. 51, 755 (1983); V. A. Kostelecký and M. M. Nieto, ibid. 53, 2285 (1984); Phys. Rev. A 32, 1293 (1985); 32, 3243 (1985).

${ }^{16} \mathrm{M}$. de Crombrugghe and V. Rittenberg, Ann. Phys. (N.Y.) 151, 99 (1983).

${ }^{17}$ There is a relationship between this system and the nonrelativistic harmonic oscillator. See R. Jackiw, Phys. Rev. D 29, 2375 (1984).

${ }^{18}$ Y. N. Srivastava and A. Widom, Lett. Nuovo Cimento 39, 285 (1984); M. Hirayama and T. Torii, Prog. Theor. Phys. 68, 1354 (1982); M. Hirayama, ibid. 70, 1444 (1983); H. Ui, ibid. 72, 266 (1984); 72, 812 (1984); A. Niemi, IAS report 1984 (unpublished); R. Gamonal, Phys. Rev. B 32, 2846 (1985).

${ }^{19}$ R. J. Hughes, V. A. Kostelecký, and M. M. Nieto, Phys. Lett. 171B, 226 (1986).

${ }^{20}$ R. P. Feynman, in High Energy Nuclear Physics, edited by G. Ascoli et al. (Interscience, New York, 1957), p. IX-42.

${ }^{21}$ R. P. Feynman and M. Gell-Mann, Phys. Rev. 109, 193 (1958).

${ }^{22}$ L. Landau and E. M. Lifshitz, Quantum Mechanics (Pergamon, London, 1973).

${ }^{23}$ A. Dupré, Am. J. Phys. 49, 34 (1981).

${ }^{24}$ In the definition (3.8), we have fixed some arbitrary signs, which establish the form of Eq. (3.11). Other equivalent expressions are possible.

${ }^{25}$ L. L. Foldy and S. A. Wouthuysen, Phys. Rev. 78, 29 (1950); W.-Y. Tsai, Phys. Rev. D 7, 1945 (1973).

${ }^{26}$ Although we believe that in principle a broken supersymmetry may be realized for the case $k_{z} \neq 0$, the construction is technically difficult. 
${ }^{27}$ W. Herbst, Commun. Math. Phys. 53, 285 (1977).

${ }^{28}$ J. R. Klauder and B.-S. Skagerstam, Coherent States, Application in Physics and Mathematical Physics (World Scientific, Singapore, 1985).

${ }^{29} \mathrm{~K}$. Johnson, in Proceedings of the Santa Fe Meeting of the
Division of Particles and Fields of the American Physical Society, 1984, edited by T. Goldman and M. M. Nieto (World Scientific, Singapore, 1985), p. 343.

${ }^{30}$ V. A. Kostelecký, M. M. Nieto, and D. R. Truax, Phys. Rev. D 32, 2627 (1985). 